\title{
Variations spatiales et temporelles de la richesse et de l'abondance des rotifères (Brachionidae et Trichocercidae) et des cladocères dans un petit lac artificiel eutrophe situé en zone tropicale Spatial and temporal variations in the richness and abundance of rotifer (Brachionidae \& Trichocercidae) and cladoceran communities in a small artifial eutrophic tropical lake
}

\author{
S. H. Zébazé Togouet, T. Njiné, N. Kemka, M. Nola, S. Foto Menbohan, A. \\ Monkiedje, D. Niyitegka, T. Simke-Ngando et L. B. Jugnia
}

Volume 18, numéro 4, 2005

URI : https://id.erudit.org/iderudit/705569ar

DOI : https://doi.org/10.7202/705569ar

Aller au sommaire du numéro

Éditeur(s)

Université du Québec - INRS-Eau, Terre et Environnement (INRS-ETE)

ISSN

0992-7158 (imprimé)

1718-8598 (numérique)

Découvrir la revue

Citer cet article

Zébazé Togouet, S. H., Njiné, T., Kemka, N., Nola, M., Foto Menbohan, S., Monkiedje, A., Niyitegka, D., Simke-Ngando, T. \& Jugnia, L. B. (2005). Variations spatiales et temporelles de la richesse et de l'abondance des rotifères (Brachionidae et Trichocercidae) et des cladocères dans un petit lac artificiel eutrophe situé en zone tropicale. Revue des sciences de l'eau / Journal of Water Science, 18(4), 485-505. https://doi.org/10.7202/705569ar
Résumé de l'article

Les communautés de rotifères (Brachionidae et Trichocercidae) et de cladocères peuplant les eaux du lac Municipal de Yaoundé situé en zone tropicale (Cameroun), ont été examinés en relation avec quelques variables physico-chimiques du milieu. Des 26 espèces et sous-espèces de rotifères (Brachionidae et Trichocercidae) et 15 espèces de cladocères inventoriées, environ 16 sont identifiées pour la première fois au Cameroun. Parmi les espèces quantitativement dominantes, les espèces fréquemment rencontrées sont Brachionus angularis angularis, $B$. falcatus falcatus, et $B$. calyciflorus chez les Brachionidae, Trichocerca elongata elongata, et $T$. bicristata bicristata chez les Trichocercidae, et Ceriodaphnia cornuta et Chydorus eurynotus chez les cladocères. Plus de $75 \%$ des espèces répertoriées sont des espèces périphytiques communément considérées comme littorales, dont le développement important a été observé dans la zone pélagique du lac qui, sans doute, offre de nombreuses ressources et constitue un refuge vis-à-vis notamment de la prédation. L'absence de corrélations entre les communautés zooplanctoniques étudiés et les quelques variables physico-chimiques suivies, nous a amenés à considérer que le développement de ces communautés est sous la dépendance d'autres facteurs. D'ailleurs, une analyse canonique de correspondance indique que les stations, profondeurs et mois de prélèvement expliqueraient respectivement $3 \%, 5 \%$ et $36 \%$ de la variance totale associée à l'abondance des espèces prises en compte. Ce qui nous a permis d'émettre l'hypothèse selon laquelle les principales conditions environnementales qui affectent le développement potentiel des espèces analysées et le déroulement de leurs cycles biologiques, seraient dépendantes des deux saisons (la saison des pluies et la saison sèche) caractérisant les climats tropicaux. 


\title{
Variations spatiales et temporelles de la richesse et de l'abondance des rotifères (Brachionidae et Trichocercidae) et des cladocères dans un petit lac artificiel eutrophe situé en zone tropicale
}

\author{
Spatial and temporal variations in the richness and \\ abundance of rotifer (Brachionidae and Trichocercidae) \\ and cladoceran communities in a small artificial \\ eutrophic tropical lake
}

\author{
S.H. ZÉBAZÉ TOGOUET ${ }^{1 *}$, T. NJINÉ ${ }^{1}$, N. KEMKA ${ }^{1}$, M. NOLA ${ }^{1}$, \\ S. FOTO MENBOHAN ${ }^{1}$, A. MONKIEDJE ${ }^{1}$, D. NIYITEGEKA ${ }^{1}$, T. SIME-NGANDO ${ }^{2}$, \\ L.B. JUGNIA
}

Reçu le 25 février 2004, accepté le 23 décembre 2004**

SUMMARY

The rotifer and cladoceran communities of a small tropical eutrophic lake (the municipal lake of Yaoundé, Cameroon) were examined from November 1996 to December 1997, in relation to some physical and chemical variables. Using a Van Dorn bottle, water samples were collected weekly from 5 depths $(0 \mathrm{~m}, 0.5 \mathrm{~m}, 1 \mathrm{~m}, 1.5 \mathrm{~m}$ and $2.5 \mathrm{~m})$ at three stations (I, II and III) representing the upper, middle and lower parts of the lake, respectively. Water temperature was measured with a thermometer placed in the sampling bottle and $\mathrm{pH}$ was measured in the field with a portable pH-meter. Oxygen concentrations were determined according to the Winkler method. Water colour, suspended solids, and ammonium-nitrogen $\left(\mathrm{NH}_{4}-\mathrm{N}\right)$ were analyzed spectrophotometrically using standard methods. The transparency of the water column was determined with a Secchi disk. Zooplankton species were identified alive under a phase contrast microscope using several manuals, and rotifers and cladocerans were counted in a Dolfuss chamber.

Throughout the study, temperature and $\mathrm{pH}$ values of the water remained around $25^{\circ} \mathrm{C}$ and 7 , respectively. The Secchi disk transparency did not exceed $1 \mathrm{~m}$ and the dissolved oxygen concentration decreased with depth to almost zero in the bottom waters. Spatial and temporal variations of these variables indicated that

1. Université de Yaoundé I, Laboratoire de Biologie Générale, BP 812 Yaoundé, Cameroun.

2. Université Blaise-Pascal Clermont-Ferrand 2, Laboratoire de Biologie des Protistes, UMR CNRS 6023 Les Cézeaux, 63177 Aubière Cedex, France.

3. Conseil National de Recherches du Canada, Institut de Recherche en Biotechnologie, 6100, avenue Royalmount, Montréal, Québec, H4P 2R2, Canada.

* Correspondance: tél. : + 2377453020 ; Fax : + 2372201854 - Email : zebaschu@yahoo.fr

** Les commentaires seront reçus jusqu'au 30 août 2006. 
the middle zone ( 0.5 and $1.5 \mathrm{~m}$ ) was the stratum with a critical role in the functioning of the lake. Indeed, it represented the transition zone between the trophogenic upper water layer $(0$ to $0.5 \mathrm{~m})$ where photosynthetic activity occurred and the bottom water layer $(1.5$ to $2.5 \mathrm{~m})$ formed by the microaerophilic to anaerobic tropholitic layer where reducing processes were important.

The rotifer and cladoceran communities were relatively diverse, with 41 species collected during the study year (i.e., 26 species of Brachionidae and Trichocercidae and 15 species of Cladocera), of which 16 are reported for the first time in Cameroon. These organisms filter small organic particles (fresh algal detritus and bacteria) and their presence in the lake could be related to the relatively high algal biomass found there. In terms of relative abundance, rotifers appear to be more important than cladocerans. Among the quantitatively dominant species, the most frequent ones were the Brachionidae ( $\mathrm{Bra}$ chionus angularis angularis, B. falcatus falcatus, B. calyciflorus), the Trichocercidae (Trichocerca elongata elongata and T. bicristata bicristata) and the Cladocera (Ceriodaphnia cornuta and Chydorus eurynotus).

Brachionus angularis was the most abundant and frequent rotifer observed during this study. According to several authors, a high abundance of Brachionus can be considered as a biological indicator of more eutrophic waters. This corroborates the hypereutrophic status assigned to the lake through previous studies dealing with bacterioplankton and phytoplankton in this system. Among Cladocera, Daphnia, an important bacterivore, was absent despite the high density of bacteria $\left(\sim 10^{8} \mathrm{cell} \cdot \mathrm{mL}^{-1}\right)$ in the lake. Previous studies in other Cameroonian aquatic systems reported the scarcity of Daphnia and these earlier authors argued that it was probably replaced by smaller sized species, which in the case of the present study would be Ceriodaphnia cornuta and Moina micrura, the only representatives of Daphniidae encountered. Up to $75 \%$ of the number of species identified were periphytic species that generally develop in the littoral zone. The massive occurrence of these species in the plankton sample of the lake was related to the high particle content. Abundance generally peaked in the middle zone of the water column, likely due to the availability of resources and the general potential for such zones to provide a refuge from predation.

However, there did not appear to be any correlation between the rotifer and cladoceran communities and physico-chemical variables when the data set was examined as whole, or grouped by seasons. This suggests that the heterogeneity in species abundances was controlled by other factors, among them sampling points, depths and time of sampling. A multivariate test using canonical correspondence analysis suggested that about $3 \%$ and $5 \%$ of the variance associated with the species abundance heterogeneity could be explained by sampling point and depth, respectively. Up to $36 \%$ of this variance was attributed to the sampling months. We therefore hypothesize that the environmental conditions affecting the development and biological cycles of the studied species are highly dependent on the prevalent seasons found in a tropical zone, i.e. the rainy and the dry seasons.

Keywords: Brachionidae, Trichocercidae, Cladocera, species diversity, tropical lakes, seasonal dynamics.

\section{RÉSUMÉ}

Les communautés de rotifères (Brachionidae et Trichocercidae) et de cladocères peuplant les eaux du lac Municipal de Yaoundé situé en zone tropicale (Cameroun), ont été examinées en relation avec quelques variables physicochimiques du milieu. Des 26 espèces et sous-espèces de rotifères (Brachionidae et Trichocercidae) et 15 espèces de cladocères inventoriées, environ 16 sont identifiées pour la première fois au Cameroun. Parmi les espèces quanti- 
tativement dominantes, les espèces fréquemment rencontrées sont Brachionus angularis angularis, $B$. falcatus falcatus, et $B$. calyciflorus chez les Brachionidae, Trichocerca elongata elongata, et $T$. bicristata bicristata chez les Trichocercidae, et Ceriodaphnia cornuta et Chydorus eurynotus chez les cladocères. Plus de $\mathbf{7 5} \%$ des espèces répertoriées sont des espèces périphytiques communément considérées comme littorales, dont le développement important a été observé dans la zone pélagique du lac qui, sans doute, offre de nombreuses ressources et constitue un refuge vis-à-vis notamment de la prédation. L'absence de corrélations entre les communautés zooplanctoniques étudiées et les quelques variables physico-chimiques suivies, nous a amenés à considérer que le développement de ces communautés est sous la dépendance d'autres facteurs. D'ailleurs, une analyse canonique de correspondance indique que les stations, profondeurs et mois de prélèvement expliqueraient respectivement $3 \%, 5 \%$ et $36 \%$ de la variance totale associée à l'abondance des espèces prises en compte. Ce qui nous a permis d'émettre l'hypothèse selon laquelle les principales conditions environnementales qui affectent le développement potentiel des espèces analysées et le déroulement de leurs cycles biologiques, seraient dépendantes des deux saisons (la saison des pluies et la saison sèche) caractérisant les climats tropicaux.

Mots clés : Brachionidae, Trichocercidae, cladocères, diversité spécifique, lacs tropicaux, dynamique saisonnière.

\section{1 - INTRODUCTION}

Les rotifères et les cladocères représentent une composante importante dans la structure et le fonctionnement des écosystèmes lacustres. Par leur activité de broutage, ces organismes forment un lien trophique essentiel entre les producteurs primaires et les organismes du niveau trophique supérieur (LAIR et al., 1998, PourRIOT et FranCEZ, 1986, SHIEL, 1995). Ainsi, il a été démontré que plus de $60 \%$ de la production primaire en milieu lacustre peut être transféré aux alevins de poissons, via la seule activité herbivore des rotifères et des cladocères (HABERMAN, 1998). L'étude de ces organismes est donc nécessaire pour l'établissement des modèles de fonctionnement des systèmes aquatiques servant, notamment, à la gestion des ressources halieutiques. Nous sommes en présence de communautés biologiques qui sont, en réalité, très diversifiées (SHIEL, 1995). On peut donc supposer qu'en raison de différences interspécifiques ou de différences de comportements autoécologiques liées aux changements de l'environnement, les rotifères et les cladocères affectent différemment le cheminement de la matière et de l'énergie dans le fonctionnement des systèmes aquatiques. Une des premières démarches visant à tester cette hypothèse consiste à faire l'inventaire des espèces présentes et à étudier leur dynamique spatiale et temporelle (ARFI et PATRITI, 1987).

Cependant, peu d'études se sont penchées sur la dynamique des populations de rotifères et de cladocères dans les écosystèmes aquatiques situés en zone tropicale ou subtropicale (GOPAL et WETZEL, 1995). Au Cameroun, quelques études concernant le zooplancton métazoaire ont été menées dans différents lacs (CHIAMBENG et al., 1991, CHIAMBENG et al., 1994, CORBET et al., 1973, GREEN, 1972, GREEN et al., 1974, PourRIot, 1968, SEGERS et MERTENS, 
1997). Cependant, il s'agit, pour la plupart de ces travaux, de relevés faunistiques résultant de campagnes d'échantillonnages ponctuelles, principalement dans les lacs de cratère de la région du sud-ouest du Cameroun. Dans cette étude, nous examinons, pour la première fois, les communautés de Brachionidés, de Trichocercidés (rotifères) et de cladocères évoluant au sein du lac municipal de Yaoundé (capitale du Cameroun), ainsi que l'importance relative des variations de leurs abondances dans l'espace (vertical et horizontal) par rapport à celles dans le temps (saisons), ceci en relation avec quelques variables physico-chimiques du milieu.

\section{2 - MATÉRIEL ET MÉTHODES}

\subsection{Site d'étude}

Le lac municipal de Yaoundé $\left(3^{\circ} 51^{\prime} 37^{\prime \prime} \mathrm{N}-11^{\circ} 30^{\prime} 40^{\prime \prime} \mathrm{E}\right)$ est un lac artificiel créé en 1951, par barrage de la rivière Mingoa. Il est situé au cœur de Yaoundé à $711 \mathrm{~m}$ d'altitude et son bassin versant d'environ $80 \mathrm{~km}^{2}$ (BOPDA, 1986) est entièrement occupé par des immeubles et des habitations. II s'agit d'un lac de petite taille (surface $=6,5 \mathrm{ha}$ ), peu profond $\left(Z_{\max }=4 \mathrm{~m}\right.$ ) recevant des eaux usées d'origine domestique (JUGNIA et SIME-NGANDO, 2001). Les concentrations en phosphore total dans le lac municipal de Yaoundé varient de 10 à $1650 \mu \mathrm{g} . \mathrm{l}^{-1}$ (KEMKA et al., 2003), les teneurs en chlorophylle a sur la base d'une étude nycthémérale se situent entre 25,3 et $234 \mu \mathrm{g} . \mathrm{I}^{-1}$, pour des effectifs d'algues (essentiellement des cyanobactéries) compris entre 1,42 à 11,73 x $10^{8}$ cellules. I $^{-1}$ (TADONLÉKÉ et al., 1998).

Dans la région du lac municipal de Yaoundé, le climat est de type sub-équatorial qualifié de yaoundéen (SUCHEL, 1987) avec quatre saisons: une grande saison sèche (mi-novembre - février), une petite saison des pluies (mars - miavril), une petite saison sèche (mi-avril - mai), une grande saison des pluies (juin - mi-novembre). Les précipitations y sont modérées (moyenne annuelle 1576 $\mathrm{mm}$ ) et la température varie peu dans le temps (moyenne annuelle $=24 \pm 2,5^{\circ} \mathrm{C}$ ).

\section{2 Échantillonnage}

Les échantillons ont été prélevés de novembre 1996 à décembre 1997 sur une fréquence hebdomadaire, dans la zone pélagique du lac et au niveau de I'herbier littoral. Dans la zone pélagique, trois stations ont été échantillonnées, la station I située en amont du lac, la station II dans la partie centrale du lac, et la station III au niveau de l'exutoire. Les échantillons ont été prélevés à $0 \mathrm{~m}, 0,5$ $\mathrm{m}, 1,0 \mathrm{~m}, 1,5 \mathrm{~m}$ et $2,5 \mathrm{~m}$ de profondeur. L'échantillonnage pélagique a été effectué à l'aide d'une bouteille de prélèvement VAN DORN de 6 litres. La station IV située au niveau de l'herbier littoral du lac a été échantillonnée manuellement à l'aide d'un récipient de verre et après agitation et pression de l'herbier pour une étude qualitative des peuplements zooplanctoniques présents. Les organismes échantillonnés et contenus dans 6 litres d'échantillons sont concentrés à l'aide d'un filet nitex de $34 \mu \mathrm{m}$ de vide de maille dans $500 \mathrm{ml}$ de sous-échantillon. Une première moitié de ce sous-échantillon est fixée au formaldéhyde boraté (concentration finale $=4 \%$ vol./vol., à partir d'une solution commerciale à $37 \%$ ) pour les dénombrements, tandis que la seconde moitié est utilisée pour les observations sur le vivant. 


\subsection{Variables abiotiques}

La température, la transparence de l'eau et le $\mathrm{pH}$ ont été mesurés in situ respectivement au moyen d'un thermomètre à mercure fixé à l'intérieur de la bouteille d'échantillonnage, d'un disque de Secchi (diamètre $=30 \mathrm{~cm}$ ) et d'un pH-mètre portable (Shott C.G.818). L'oxygène dissous a été mesuré par la méthode de Winkler (MACKERETH et al., 1978). La couleur de l'eau, les teneurs en matières en suspension (MES) et l'azote ammoniacal ont été mesurés par spectrophotométrie (spectrophotomètre HACH DR/2000) selon les méthodes APHA (1985). Les variables abiotiques ont été, en général, mesurées uniquement au niveau de la station pélagique II située dans la partie centrale du lac.

\subsection{Variables biotiques}

De retour au laboratoire, les échantillons zooplanctoniques sont placés dans des cuves de DOLFUSS avant examen, selon les recommandations de LEGENDRE et WATT (1972). Les dénombrements et l'identification des individus sont ensuite effectués sous une loupe binoculaire (WILD M5) ou un microscope à contraste de phase (Leitz Orthoplan). L'identification des rotifères se réfère aux clés de détermination de KOSTE (1978), POURRIOT et FRANCEZ (1986) et SHIEL (1995). Celle des cladocères se réfère aux ouvrages d'identification de AMOROS (1984), SMIRNOV et KOROVCHINSKY (1995) et SMIRNOV (1996).

\subsection{Statistique}

Les analyses statistiques ont été effectuées à partir des données brutes recueillies sur une fréquence hebdomadaire. Les relations entre variables ont été établies à l'aide de l'analyse statistique de corrélation de rang de Spearmann corrigée selon l'approche de Bonferroni. Afin de discriminer l'influence des différentes stations, profondeurs et mois de prélèvements sur l'hétérogénéité de l'abondance des communautés zooplanctoniques, les données de dénombrements zooplanctoniques ont été croisées avec les facteurs spatiotemporels par une analyse canonique des correspondances (ACC) (logiciel ADE-4, (THIOULOUSE et al., 1997)). Cette analyse permet de croiser les tableaux des variables spatiales ( 3 stations $\times 5$ profondeurs), temporelles ( 3 stations $\times$ 5 profondeurs $\times 12$ mois) avec celui des dénombrements zooplanctoniques (12 espèces $\times 870$ dénombrements). Ainsi, trois analyses canoniques de correspondances ont été effectuées, à raison d'une pour chaque facteur de variation (station, profondeurs, mois).

\section{3 - RÉSULTATS}

\subsection{Environnement physico-chimique}

Au cours de l'étude, la température de la colonne d'eau a varié relativement peu, avec une amplitude de fluctuation de $\pm 2,5^{\circ} \mathrm{C}$ autour de $25^{\circ} \mathrm{C}$. Un refroidissement de la colonne d'eau est observé entre les mois d'avril et juillet (figure $1 \mathrm{~A}$ ). Les valeurs $\mathrm{du} \mathrm{pH}$ ont également fluctué relativement peu, de $\pm 0,5$ unité autour de 7 (figure $1 \mathrm{G}$ ). 

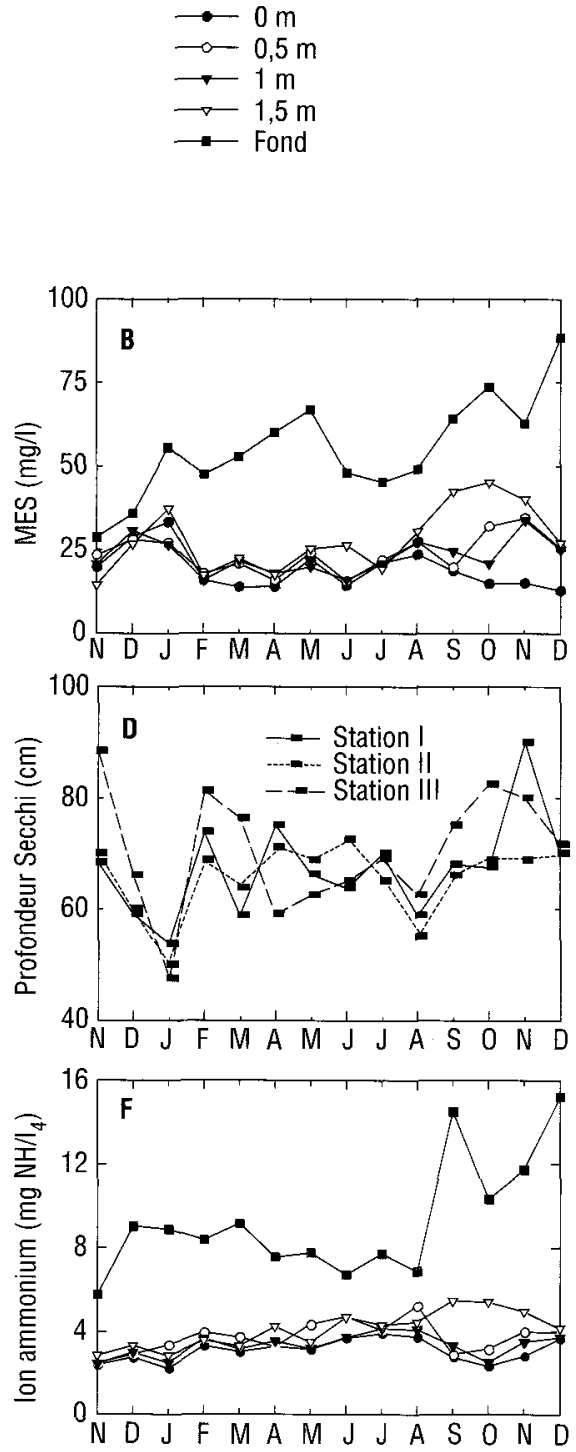
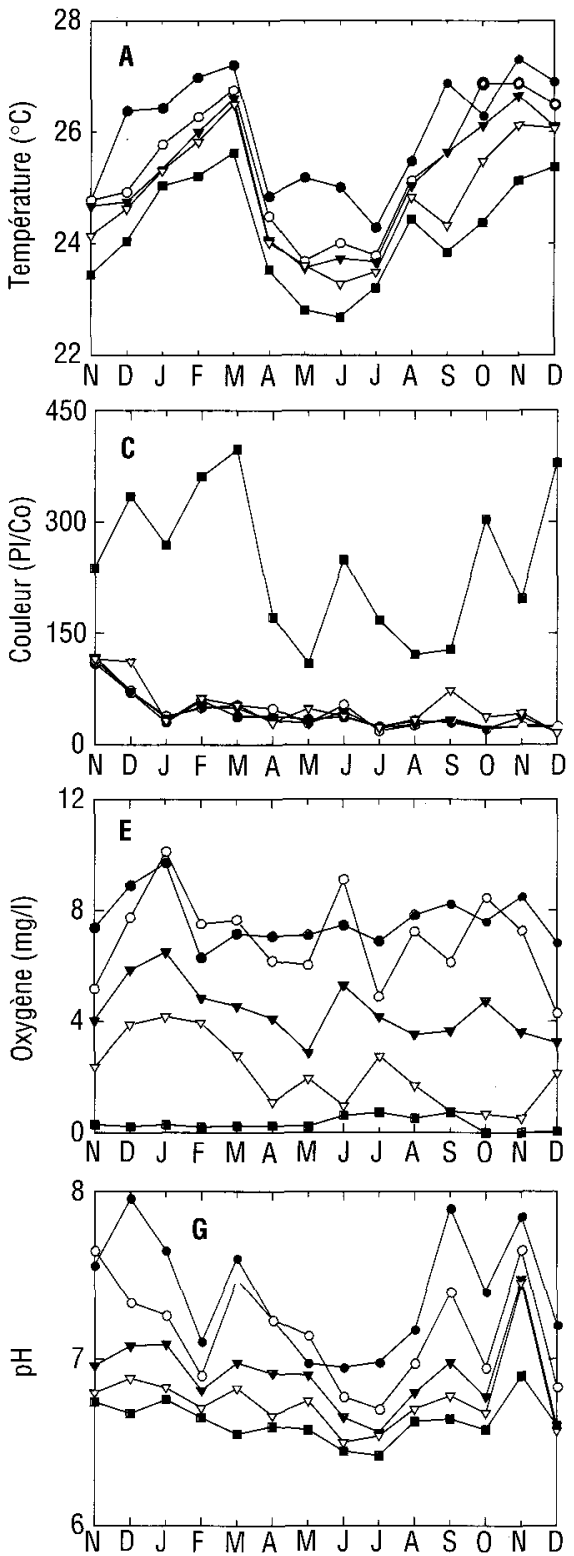

Figure 1 Variations verticales et saisonnières de quelques variables physicochimiques mesurées à la station II située dans la partie centrale la plus profonde du lac municipal de Yaoundé (Cameroun). Les valeurs mensuelles représentent des valeurs moyennes obtenues à partir d'un échantillonnage hebdomadaire.

Vertical and seasonal variations in the physico-chemical variables measured at station /I located at the deepest central region in the municipal lake Yaoundé (Cameroon). Data for each month represent the mean value of weekly sampling. 
Les valeurs des teneurs en MES ainsi que de la couleur des eaux, sont nettement plus élevées en profondeur qu'en surface (figure $1 \mathrm{~B}, \mathrm{C}$ ). De manière générale, les eaux du lac municipal de Yaoundé sont colorées et chargées en MES. La profondeur de disparition du disque de Secchi est restée inférieure à $1 \mathrm{~m}$ au cours de l'étude, au niveau des trois stations pélagiques échantillonnées (figure $1 \mathrm{D}$ ). Les concentrations en oxygène dissous baissent avec la profondeur. En effet, les valeurs sont comprises entre 4,1 et $18,3 \mathrm{mg} / \mathrm{l}$ en surface, alors que des conditions plutôt anoxiques prévalent dans les couches inférieures du lac, notamment au-delà de $1,5 \mathrm{~m}$ de profondeur où les valeurs ont fluctué entre 0 et $0,7 \mathrm{mg} / \mathrm{l}$ (figure $1 \mathrm{E}$ ). Les teneurs en azote ammoniacal sont plus élevées dans les eaux profondes qu'en surface (figure $1 \mathrm{~F}$ ).

\subsection{Composition spécifique des peuplements étudiés}

\subsubsection{Rotifères : Brachionidae et Trichocercidae (tableau 1)}

Les Brachionidae sont représentées par 15 espèces et sous-espèces appartenant aux genres Anuraeopsis, Brachionus, Notholca, Keratella et Platyias. De ces espèces, Anuraeopsis fissa, Brachionus angularis angularis, B. falcatus falcatus et Keratella tropica sont les plus fréquentes et les plus abondantes (tableau 1). Six des espèces de Brachionidés du lac sont connues comme des espèces essentiellement pélagiques: Anuraeopsis fissa, Brachionus angularis angularis, $B$. calyciflorus anuraeiformis, $B$. falcatus falcatus, $B$. leydigi leydigi et Keratella tropica. Ces espèces ont été récoltées dans toutes nos stations, y compris dans l'herbier littoral. L'espèce Notholca labis n'a été collectée qu'à la station I. Une seule espèce, Anuraeopsis navicula, est apparue inféodée à l'herbier littoral du lac.

En ce qui concerne les Trichocercidae, 11 espèces et sous-espèces ont pu être identifiées, dont 10 appartenant au genre Trichocerca. En dehors de deux espèces (Trichocerca dixon-nutalli et $T$. insignis) qui n'ont été rencontrées que dans la zone pélagique, tous les autres représentants des Trichocercidés se développent en eau libre et au niveau de l'herbier littoral, y compris $T$. elongata connue comme espèce essentiellement pélagique (tableau 1).

\subsubsection{Cladocères (tableau 2)}

Nous avons pu identifier 15 espèces et sous-espèces de Cladocères appartenant à cinq familles différentes (Sididae, Daphniidae, Macrothricidae, Ilyocryptidae et Chydoridae) (tableau 2). Ces espèces sont rares et généralement littorales et périphytiques. Seules les espèces Ceriodaphnia cornuta et, plus rarement, Chydorus eurynotus, ont été collectées aux trois stations pélagiques visitées. Il est à noter l'absence du genre Daphnia au cours de cette étude, Ceriodaphnia cornuta et Moina micrura étant les seuls représentants des Daphniidae observés au sein du lac municipal de Yaoundé (tableau 2). 
Tableau 1 Liste et fréquence d'apparition [(nombre d'échantillons contenant l'espèce/nombre total d'échantillons) $\times$ 100] des espèces de Rotifères échantillonnées dans le lac municipal de Yaoundé (Cameroun) entre novembre 1996 et décembre 1997.

Table 1 Appearance frequency (Number of samples containing the specie/total number of samples) $\times 100$ ) and list of rotiferans sampled in the municipal Lake of Yaoundé (Cameroon) between November 1996 and December 1997.

\begin{tabular}{|c|c|c|c|c|c|c|c|}
\hline \multirow{2}{*}{ Sous-classe } & \multirow{2}{*}{ Ordre } & \multirow{2}{*}{ Famille } & \multirow{2}{*}{ Espèce } & \multicolumn{4}{|c|}{ Fréquence (\%) par station échantillonnée } \\
\hline & & & & & II & III & Herbier \\
\hline \multirow{26}{*}{$\begin{array}{l}\text { Eurotatoria } \\
\text { (Super ordre } \\
\text { Digononta) }\end{array}$} & \multirow[t]{26}{*}{ Ploimida } & \multirow{15}{*}{$\begin{array}{l}\text { Brachionidae } \\
\text { Wesenberg-Lund, } \\
1899\end{array}$} & Anuraeopsis fissa Gosse, 1851 & 100 & 100 & 100 & 100 \\
\hline & & & A. navicula Rousselet, 1910 & 0 & 0 & 0 & 3,5 \\
\hline & & & Brachionus angularis angularis (Gosse, 1851) & 100 & 100 & 100 & 93 \\
\hline & & & B. calyciflorus Pallas, 1766 & 33 & 28 & 29 & 38 \\
\hline & & & B. calyciflorus anuraeiformis (Brehm, 1909) & 2 & 0 & 0 & 5 \\
\hline & & & B. falcatus falcatus Zacharias, 1898 & 88 & 90 & 90 & 72,5 \\
\hline & & & B. leydigi leydigi Cohn, 1862 & 14 & 10,5 & 9 & 26 \\
\hline & & & B. leydigi rotundus (Rousselet, 1907) & 4 & 0 & 0 & 7 \\
\hline & & & B. patulus (OF. Müller, 1786) & 2 & 2 & 2 & 22,5 \\
\hline & & & B. quadridentatus quadridentatus (Hermann, 1783) & 7 & $3, \overline{5}$ & 5 & 38 \\
\hline & & & Brachionus sp. & 0 & 0 & 2 & 3,5 \\
\hline & & & Keratella tropica (Apstein, 1907) & 95 & 98 & 95 & 65,5 \\
\hline & & & Notholca labis Gosse, 1887 & 3,5 & 0 & 0 & 0 \\
\hline & & & Platyias leloupi leloupi (Gillard, 1957) & 2 & 0 & 0 & 26 \\
\hline & & & P. quadricornis (Ehrenberg, 1832) & 2 & 0 & 0 & 67 \\
\hline & & \multirow{11}{*}{$\begin{array}{l}\text { Trichocercidae } \\
\text { Remane, } 1933\end{array}$} & Trichocerca (s str) bicristata bicristata (Gosse, 1887) & 36 & 50 & 34,5 & 15,5 \\
\hline & & & T.(D) dixon-nuttalli Jennings, 1903 & 0 & 5 & 2 & 0 \\
\hline & & & T.(s. str) elongata elongata (Gosse, 1886) & 48 & 62 & 50 & 36 \\
\hline & & & T.(s. str) elongata tchadiensis (Pourriot, 1968) & 5 & 3,5 & 2 & 5 \\
\hline & & & T.(D) insignis (Herrick, 1885) & 0 & 0 & 2 & 0 \\
\hline & & & T.(D) pusilla (Lauterborn, 1895) & 0 & 0 & 0 & 12 \\
\hline & & & T.(s. str) rousseleti (Voigt, 1902) & 14 & 14 & 10 & 7 \\
\hline & & & T.(s. str) stylata (Gosse, 1851) & 38 & 43 & 36 & 19 \\
\hline & & & T. (D) tenuior (Gosse, 1886) & 0 & 0 & 0 & 2 \\
\hline & & & T.(D) tigris (0.F. Müller, 1786) & 0 & 0 & 0 & 2 \\
\hline & & & Trichocerca sp. & 2 & 0 & 2 & 7 \\
\hline
\end{tabular}


Tableau 2 Liste et fréquence d'apparition [(nombre d'échantillons contenant l'espèce/nombre total d'échantillons) $\times 100$ ] des espèces de Cladocères échantillonnées dans le lac municipal de Yaoundé (Cameroun) entre novembre 1996 et décembre 1997.

Table 2 Appearance frequency (Number of samples containing the specie/total number of samples) $\times 100$ ) and list of cladocerans sampled in the municipal Lake of Yaoundé (Cameroon) between November 1996 and December 1997.

\begin{tabular}{|c|c|c|c|c|c|c|c|}
\hline \multirow{2}{*}{ Classe } & \multirow{2}{*}{ Ordre } & \multirow{2}{*}{ Famille } & \multirow{2}{*}{ Espèce } & \multicolumn{4}{|c|}{ Fréquence $(\%)$ par slation échantillonnée } \\
\hline & & & & & II & III & Herbier \\
\hline \multirow[t]{5}{*}{$\begin{array}{l}\text { Branchiopoda } \\
\text { Latreille, } 1817\end{array}$} & Ctenopoda (Sars, 1865) & Sididae (Baird, 1850) & $\begin{array}{l}\text { Diaphanosoma volzi (Stingelin, 1905) } \\
\text { Pseudosida bidentata (Herrick, 1884) } \\
\text { P. szalayi (Daday, 1898) }\end{array}$ & $\begin{array}{l}3,5 \\
2 \\
0\end{array}$ & $\begin{array}{l}0 \\
2 \\
0\end{array}$ & $\begin{array}{l}0 \\
2 \\
0\end{array}$ & $\begin{array}{r}19 \\
12 \\
3,5\end{array}$ \\
\hline & Anomopoda (Sars, 1865) & Daphniidae (Straus, 1820) & $\begin{array}{l}\text { Ceriodaphnia cornuta (Sars, 1886) } \\
\text { Moina micrura (Kurz, 1874) }\end{array}$ & $\begin{array}{c}22,5 \\
2\end{array}$ & $\begin{array}{r}26 \\
3,5\end{array}$ & $\begin{array}{r}26 \\
0\end{array}$ & $\begin{array}{r}50 \\
7\end{array}$ \\
\hline & & $\begin{array}{l}\text { Macrothricidae (Norman } \\
\text { et Brady, 1867) }\end{array}$ & $\begin{array}{l}\text { Guernella raphaelis (Richards, 1892) } \\
\text { Macrothrix laticornis (Jurine, } 1820 \text { ) }\end{array}$ & $\begin{array}{l}0 \\
0\end{array}$ & $\begin{array}{l}2 \\
0\end{array}$ & $\begin{array}{l}0 \\
0\end{array}$ & $\begin{array}{l}34,5 \\
15,5\end{array}$ \\
\hline & & Ilyocryptidae (Smirnov, 1992) & $\begin{array}{l}\text { Ilyocryptus sordidus (Lievin, 1848) } \\
\text { l. halyi (Smirnov, 1992) } \\
\text { l. spinifer (Herrick, 1882) }\end{array}$ & $\begin{array}{l}5 \\
0 \\
0\end{array}$ & $\begin{array}{l}0 \\
0 \\
2\end{array}$ & $\begin{array}{l}0 \\
0 \\
2\end{array}$ & $\begin{array}{l}5 \\
5 \\
5\end{array}$ \\
\hline & & Chydoridae (Stebbing, 1902) & $\begin{array}{l}\text { Alona costata (Sars, 1862) } \\
\text { A. guttata (Sars, } 1862 \text { ) } \\
\text { A. guttata rectangularis (Sars, 1862) } \\
\text { Chydorus eurynotus (Sars, 1901) } \\
\text { Kurzia longirostris (Daday, 1898) }\end{array}$ & $\begin{array}{l}3,5 \\
0 \\
0 \\
7 \\
0\end{array}$ & $\begin{array}{r}0 \\
0 \\
0 \\
3,5 \\
0\end{array}$ & $\begin{array}{l}0 \\
0 \\
0 \\
2 \\
0\end{array}$ & $\begin{array}{r}13,5 \\
24 \\
3,5 \\
26 \\
12\end{array}$ \\
\hline
\end{tabular}




\subsection{Variations spatiales et saisonnières de l'abondance des peuplements étudiés}

Les variations spatio-temporelles des espèces de rotifères et de cladocères les plus fréquentes et les plus abondantes (> 10 à 200 ind./l) au cours de notre étude, sont présentées sur la figure 2. De manière générale, les pics d'abondance ont été observés dans la couche intermédiaire du lac (entre 0,5 et $1 \mathrm{~m}$ ). L'espèce Brachionus angularis est le rotifère le plus abondant du lac municipal de Yaoundé, avec une abondance rarement en deçà de 1000 ind./l. Cette espèce se développe préférentiellement pendant la grande saison des pluies (juin-août), à toutes les stations pélagiques (figure $2 A)$. Une espèce homologue, $B$. falcatus, se développe plutôt durant les saisons sèches, entre décembre et avril (figure $2 B$ ). II en est de même pour $B$. calyciflorus dont un développement très important a été noté en février 1997 à la station III (figure 2C). Le rotifère Keratella tropica présente une variation spatio-temporelle similaire à celle de $B$. angularis, notamment aux stations I et II. Sur la station III, le développement massif de cette espèce se fait tardivement, en septembre/octobre (figure 2D).

A Brachionus angularis

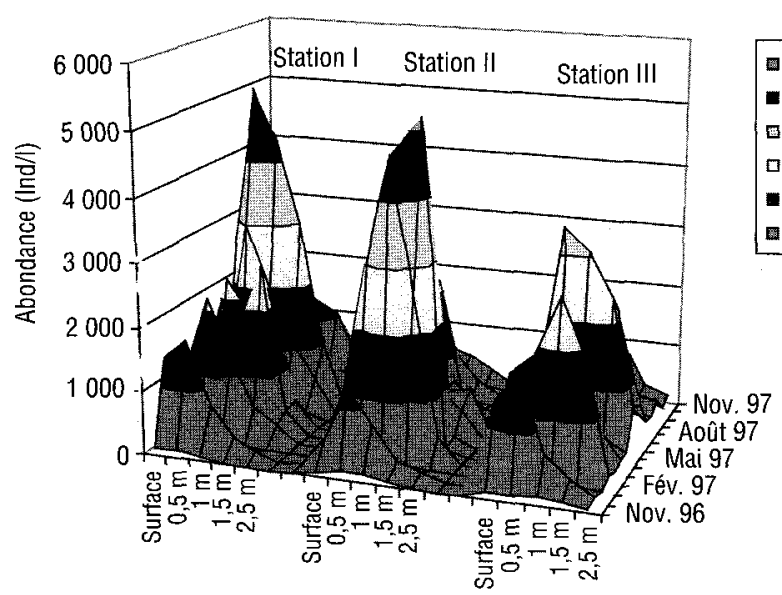

Figure 2 Variations verticales et saisonnières de l'abondances des quatre espèces de Rotifères fréquentes et quantitativement dominantes dans le lac municipal de Yaoundé (Cameroun).

Vertical and seasonal variations in the abundance of the four most frequent Rotifer species that quantitatively dominate in the municipal lake of Yaoundé (Cameroon). 

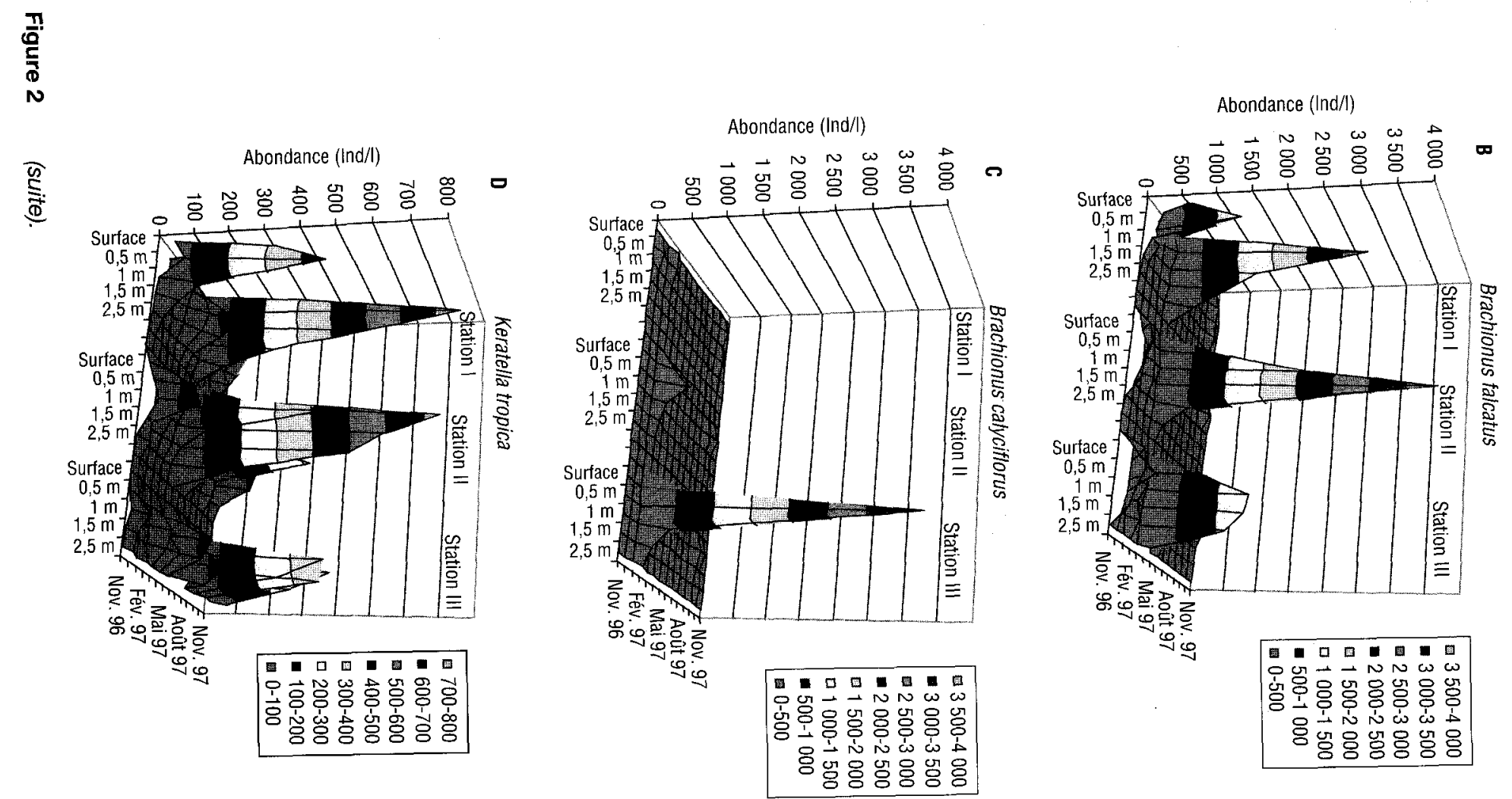
Les deux espèces les plus fréquentes et les plus abondantes du groupe des cladocères, Ceriodaphnia cornuta et Chydorus eurynotus, présentent des variations spatiales et saisonnières identiques. Ces espèces sont plus abondantes dans la couche intermédiaire du lac, comme avec les rotifères. Les variations saisonnières des deux espèces dominantes de cladocères se caractérisent par un développement important entre novembre et janvier à toutes les stations pélagiques, correspondant à la saison sèche. Pour illustrer les résultats donnés ci-dessus, les variations spatio-temporelles de Ceriodaphnia cornuta et Chydorus eurynotus sont présentées respectivement sur les figures $3 \mathrm{~A}$ et $3 \mathrm{~B}$. Entre janvier et juillet 97 , aucun développement important de cladocère n'a été noté. La seule espèce de cladocère présente en pleine eau durant cette période, Ilyocryptus sordidus, présente des densités très faibles (1 ou 2 ind./I).
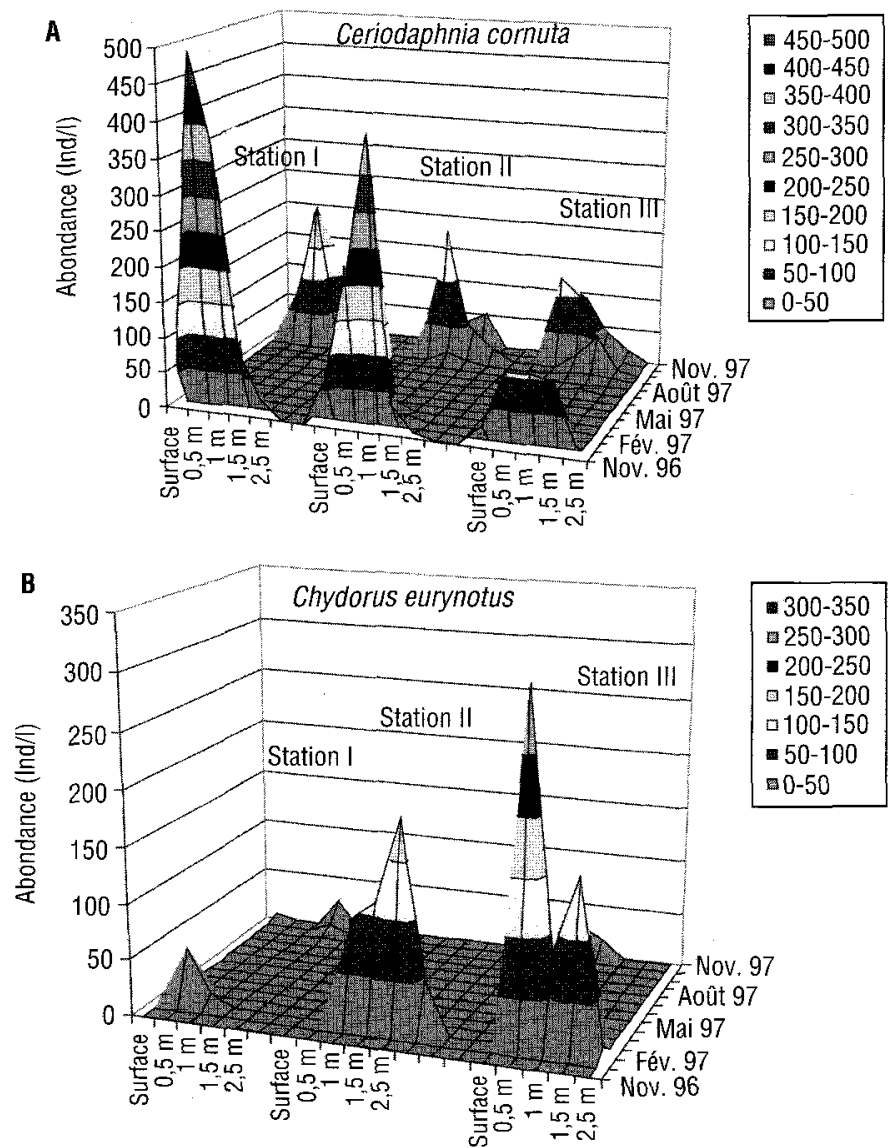

Figure 3 Variations verticales et saisonnières de l'abondance de deux espèces de Cladocères fréquentes et quantitativement dominantes dans le lac municipal de Yaoundé (Cameroun).

Vertical and seasonal variations in the abundance of the two most frequent Cladoceran species that quantitatively dominate the municipal lake of Yaoundé (Cameroon). 
Nous n'avons obtenu aucune corrélation significative entre les variables biologiques et physico-chimiques à travers l'analyse de corrélation de Spearmann corrigée selon l'approche de Bonferroni. Ceci est resté valable même en subdivisant les données en fonction des différentes saisons. Par contre, les résultats de l'analyse canonique de correspondances (ACC) visant à discriminer l'influence des différentes stations (variation horizontale), profondeurs (variation verticale) et mois (variation saisonnière) de prélèvement sur l'hétérogénéité de l'abondance des communautés zooplanctoniques étudiées, ont été significatifs $(p<0,05)$. Ces résultats indiquent que les stations et profondeurs de prélèvement expliqueraient suivant le $1^{\text {er }}$ axe des ACC (figures $4 \mathrm{~A}$ et $5 \mathrm{~A}$ ), respectivement $3 \%$ et $5 \%$ de la variance totale des effectifs zooplanctoniques, alors que plus du tiers $(36 \%)$ de cette variance serait expliqué suivant les deux premiers axes d'une autre ACC (figure $6 \mathrm{~A}$ ), par les différents mois de prélèvements.
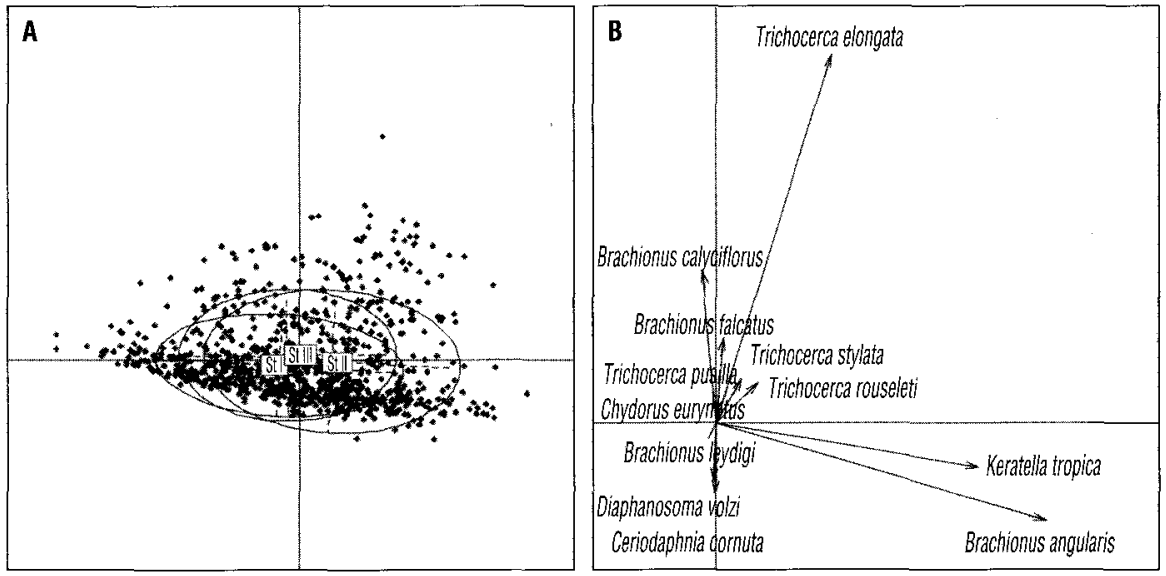

Figure 4 Analyse canonique des correspondances des différentes stations de prélèvement $(A)$ en relation avec les communautés zooplanctoniques étudiées $(B)$.

Canonical correspondence analysis of the different sampling stations (A) in relation to zooplankton communities (B).

Sur le plan horizontal, la variabilité des abondances à la station l est comparable à celle observée à la station III, et diffère de celle relevée à la station II suivant l'axe de la première composante de I'ACC (figure 4A) fortement influencé par les espèces $B$. angularis et $K$. tropica (figure 4B). II en est de même sur le plan vertical où les variations d'abondance dans les eaux profondes $(1,5$ et $2,5 \mathrm{~m})$ s'opposent à celles observées dans les eaux intermédiaires du lac $(0,5$ et $1 \mathrm{~m})$, suivant l'axe de la première composante (figure $5 \mathrm{~A}$ ) qui regroupe les espèces $B$. falcatus et $B$. angularis, $K$. tropica (figure 5B). La $3^{e}$ ACC menée sur les variables biologiques confirme l'évolution saisonnière des principales espèces décrites plus haut (figure $6 \mathrm{~A}$ et $\mathrm{B}$ ). En effet, les mois de janvier et février correspondant à la saison sèche, se distinguent suivant l'axe de la première composante regroupant les espèces $B$. calcyforus et $T$. elongata. Ces mois de la période sèche s'opposent d'une part aux mois de transition entre saison sèche et pluvieuse (avril et novembre) marqués par une 
présence apparemment plus importante de la seule espèce $B$. falcatus dans les eaux du lac, et d'autre part mais dans une moindre mesure, aux mois de juillet et août (saison des pluies) caractérisés par de fortes densités des espèces $K$. tropica et $B$. angularis. De manière générale, au cours de cette étude, le nombre total des espèces répertoriées à chacune des trois stations pélagiques prises en compte, a varié considérablement (au moins deux unités) en fonction du temps (figure 7).
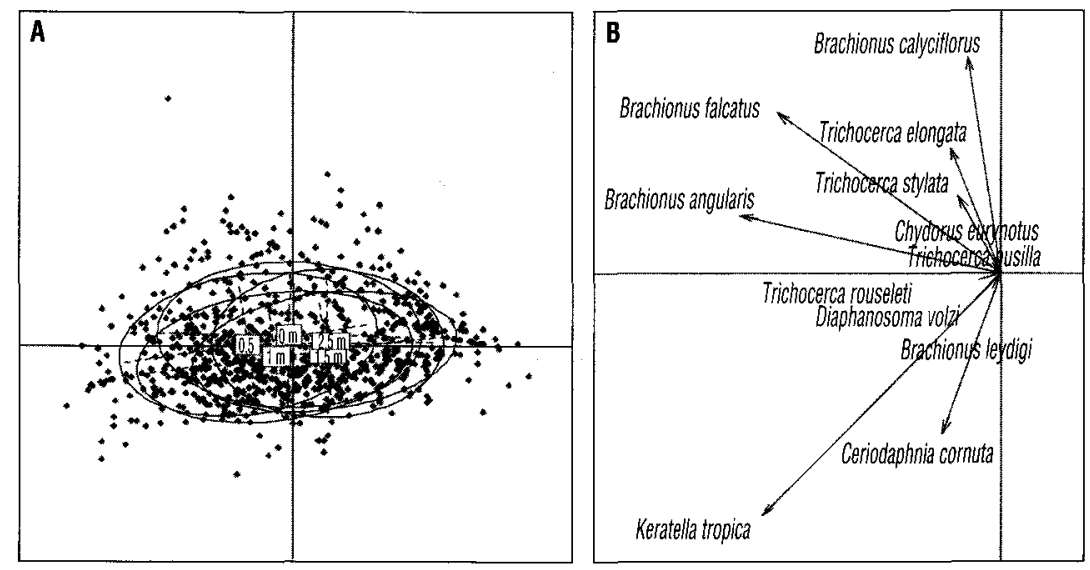

Figure $5 \quad$ Analyse canonique des correspondances des différentes profondeurs de prélèvement $(A)$ en relation avec les communautés zooplanctoniques étudiées $(B)$.

Canonical correspondence analysis of the different sampling depths $(A)$ in relation to zooplankton communities $(B)$.
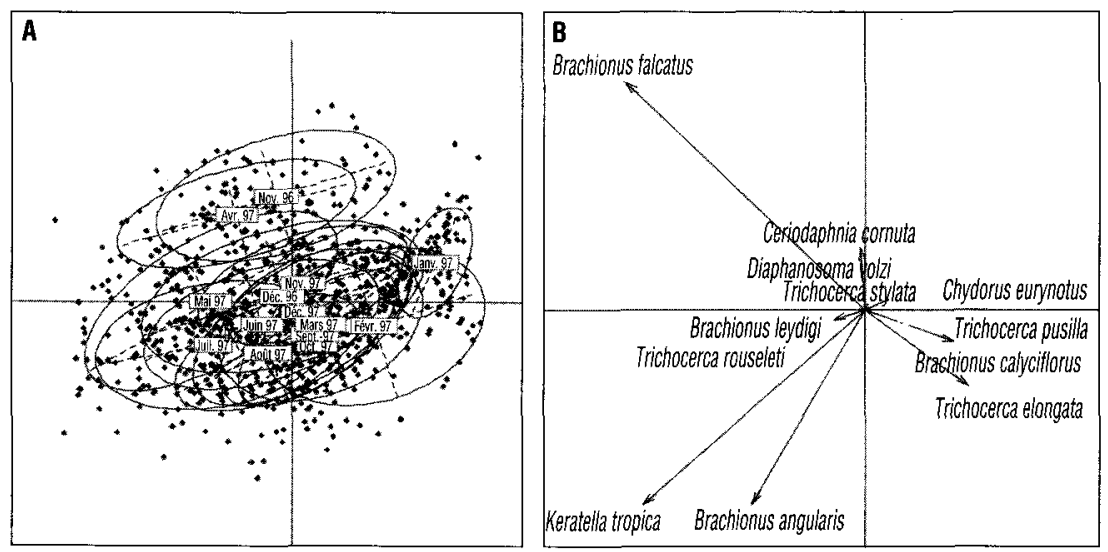

Figure 6 Analyse canonique des correspondances des différents mois de prélèvement $(A)$ en relation avec les communautés zooplanctoniques étudiées (B).

Canonical correspondence analysis of the different sampling months $(A)$ in relation to zooplankton communities $(B)$. 

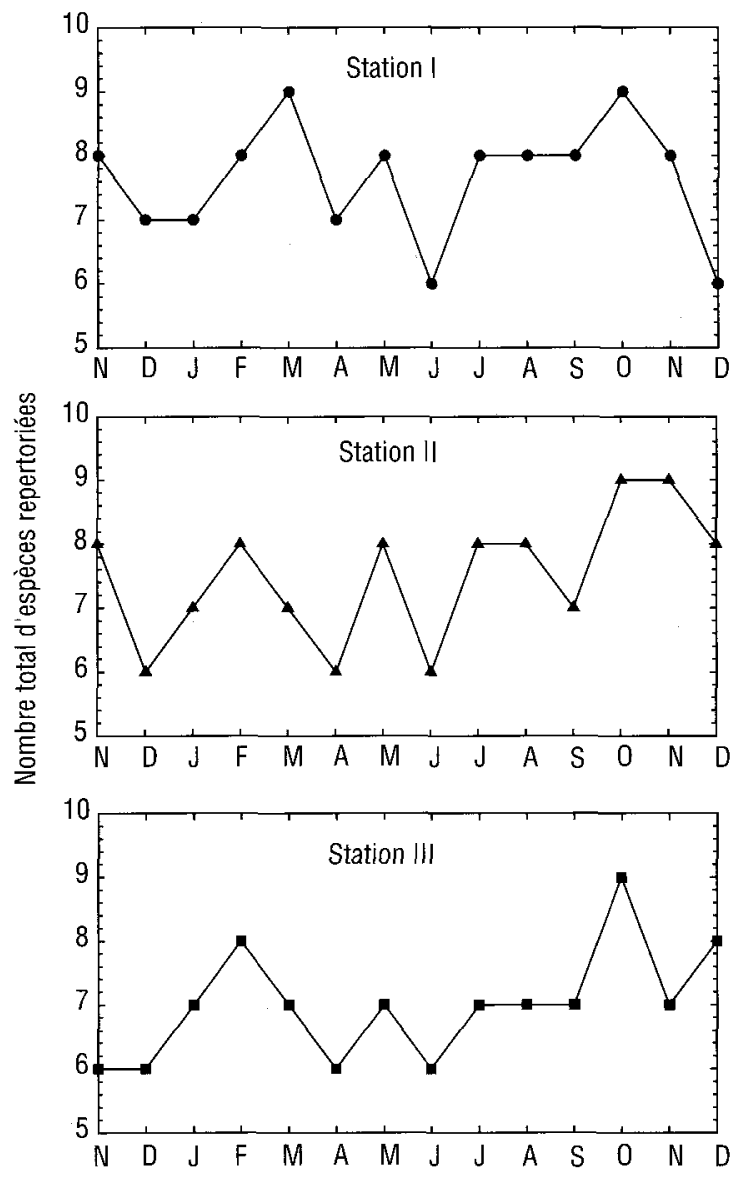

Figure $7 \quad$ Variations temporelles de la richesse spécifique totale (Cladocères + Rotifères) aux trois stations pélagiques de prélèvements (I, II et III) dans le lac municipal de Yaoundé.

Temporal variations of the total species richness (Cladocera + Rotifers) at the three pelagic sampling stations (I, II and III) within the municipal lake of Yaoundé.

\section{4 - DISCUSSION}

\subsection{Environnement physico-chimique}

Les caractéristiques physiques et chimiques des eaux du lac municipal de Yaoundé sont typiques de celles de petits lacs tropicaux peu profonds comme le lac George en Ouganda (VINER, 1969) ou les lacs du Nigeria, Oguta (NWADIARO, 1987) et OPA (AKIMBUWA et ADENIYI, 1996). Ces lacs se caractérisent naturellement par la stabilité de la température des eaux et une absence de gradient thermique vertical marqué (LEWIS, 1987). Ce sont plutôt les variations verticales des paramètres tels que l'intensité lumineuse ou les teneurs en oxy- 
gène dissous ou en éléments nutritifs qui, généralement, définissent la variabilité verticale saisonnière dans ces types de lacs (ALMAZAN et BOYD, 1978, KLEIN, 1959). Dans le cadre de cette étude, ces variations indiquent que la zone $0,5-1,5 \mathrm{~m}$ constitue une strate critique dans le fonctionnement du lac municipal de Yaoundé. Cette strate constitue vraisemblablement une zone de transition entre la couche trophogène de surface où s'effectue l'essentiel de la photosynthèse, et la couche tropholytique microaérophile à anaérobique profonde, où prévaut l'oxydation respiratoire ainsi que le déroulement de l'essentiel des processus réducteurs. Cela semble être confirmé par la richesse des eaux profondes du lac en ammonium, élément nutritif généralement indicateur des processus de recyclage hétérotrophe.

\subsection{Abondance et composition spécifique}

D'un point de vue biologique, cette étude montre que des peuplements relativement diversifiés de Brachionidés, de Trichocercidés et de cladocères se développent dans le lac municipal de Yaoundé, avec 15 espèces et sousespèces de Brachionidés, 11 espèces et sous-espèces de Trichocercidés et 15 espèces et sous-espèces de cladocères (tableaux 1 et 2). Ces micro-organismes sont pour la plupart des filtreurs des particules organiques de petites tailles (détritus frais d'algues et bactéries) (BENNDORF et HENNING, 1989, DUMONT, 1977). Leur présence dans le lac municipal de Yaoundé peut donc être mise en relation avec les efflorescences de la biomasse algale relativement élevée dans ce lac (TADONLÉKÉ et al., 1998).

En termes d'abondance relative, les rotifères sont apparus plus importants que les cladocères. Cette tendance rejoint celles déjà rapportées par d'autres auteurs dans des réservoirs situés en milieu tropical (ARCIFA, 1984, BRANCO et al., 2002, DUNCAN et GULATI, 1981, KHAN et EJIKE, 1984, MASUNDIRE, 1989, MATSUMURA-TUNDISI et al., 1990, NOGUEIRA, 2001, PAGGl et JOSE DE PAGGI, 1990). Deux principaux facteurs sont évoqués pour expliquer cette supériorité numérique des rotifères au sein de ces réservoirs:

1) leur caractère opportuniste qui leur permettrait de mieux résister aux variations des conditions environnementales (MATSUMURA-TUNDISI et al., 1990), et

2) leur plus grande compétitivité dans ces milieux en raison non seulement de leur plasticité alimentaire vis-à-vis des ressources disponibles, mais aussi à cause de leur petite taille qui les rend moins vulnérables à la pression de prédation (DUMONT, 1977).

Les rotifères représentent en eaux douces le groupe animal le plus important appartenant à la niche écologique des petits filtreurs (MARGALEF, 1983, PAGGI et JOSE DE PAGGI, 1990). Les rotifères sont capables d'ingérer des particules de petite taille telles que les bactéries et détritus organiques souvent abondants dans les milieux eutrophes. II en résulte, d'après MARGALEF (1983), BLANCHER (1984), ORCUTT et PACE (1984), PACE (1986) qu'une forte représentativité des rotifères en milieu aquatique d'eau douce peut être considérée comme un indicateur biologique d'un niveau trophique élevé. Les lacs et réservoirs situés en milieu tropical sont généralement plus eutrophes que ceux en zone tempérée (LEWIS, 2000). Cela semble se confirmer car la présence du genre Brachionus est fréquente dans les eaux tropicales (BRANCO et al., 2002, 
GREEN, 1994, KHAN et EJIKE, 1984, NOGRADY, 1983). Par ailleurs, GANNON et STEMBERGER (1978), SLADECEK (1983), MAEMETS (1983), BRANCO et al. (2002) ont tous pu établir une relation entre un effectif élevé des espèces appartenant au genre Brachionus et un niveau trophique élevé. Au cours de cette étude, c'est $B$. angularis qui a été parmi les rotifères, l'espèce la plus fréquente et quantitativement dominante. Ce qui semble corroborer le statut hypereutrophe attribué au lac municipal de Yaoundé à travers les études antérieures portant sur les compartiments bactério- (JUGNIA et al., 1998) et phytoplanctonique (TADONLÉKÉ et al., 1998).

D'après les travaux de WYLIE et CURRIE (1991), 16 à $21 \%$ du carbone nécessaire aux cladocères proviendrait de leur activité de prédation sur les bactéries. Les concentrations bactériennes $\left(\sim 10^{8}\right.$ bactéries $\left.\mathrm{ml}^{-1}\right)$ dans les eaux du lac municipal de Yaoundé sont parmi les plus élevées connues en milieu aquatique (JUGNIA et al., 1998). Ce qui laisse supposer un fort développement des Daphniidae connus pour leur activité bactérivore (THOUVENOT et al., 1999). Or, nos résultats mettent en évidence l'absence du genre Daphnia dans le lac Municipal de Yaoundé. GREEN et KLING (1988) rapportaient déjà la forte rareté de ce cladocère commun en zone tempérée, dans les lacs du Cameroun. Plus récemment, DUMONT (1994) a expliqué cette rareté par le remplacement des Daphniidae de grande taille dont fait partie le genre Daphnia, par des formes de plus petite taille comme Ceriodaphnia et Moina. Au lac municipal de Yaoundé, ces espèces de substitution sont représentées par Ceriodaphnia cornuta et plus rarement, par Moina micrura (tableau 2).

L'ensemble des résultats acquis concernant la diversité des deux familles de rotifères et des cladocères dominantes dans le lac municipal de Yaoundé, met en évidence la méconnaissance de la microfaune aquatique du Cameroun (SEGERS et MERTENS, 1997). En effet, de nombreuses espèces identifiées dans le lac municipal de Yaoundé au cours de cette étude, notamment Platyias leloupi leloupi, Notholca labis, Trichocerca insignis, T. tenuior, Diaphanosoma volzi, Pseudosida szalayi, P. bidentata, Ceriodaphnia cornuta rigaudi, Moina micrura, Guernella raphaelis, Macrothrix laticornis, Ilyocryptus sordidus, I. hyali, Alona costata, Alona guttata et $A$. guttata rectangularis, n'ont jamais été rapportées auparavant dans les quelques études de nature ponctuelle effectuées jusqu'ici dans les lacs du sud-ouest du Cameroun (CHIAMBENG et al., 1991, 1994, CoRBET et al., 1973, GREEN, 1972, SEgERS et MERTENS, 1997) ou dans le lac Tchad situé en partie au nord du Cameroun (POURRIOT, 1968). II s'agit pourtant d'espèces cosmopolites des lacs et des réservoirs, évoluant donc également en zone tropicale (JOSEE DE PAGGl et KOSTE, 1995).

\subsection{Répartition spatio-temporelle}

Des 41 espèces inventoriées, 9 seulement sont des espèces reconnues pour leur mode de vie essentiellement pélagique. L'essentiel des espèces rencontrées au cours de cette étude (environ 78,1\% du total) concerne des espèces littorales et périphytiques. Au sein du lac municipal de Yaoundé, le développement plus ou moins important des espèces littorales et périphytiques peut s'expliquer par les échanges latéraux de matière entre zone pélagique et zone littorale favorisés par les facteurs physiques tels que la faible profondeur du lac et l'absence d'une stratification thermique stabilisante pour la colonne d'eau. La forte charge particulaire du lac dans la zone pélagique a été récem- 
ment liée à différentes causes potentielles: (i) la resuspension fréquente du matériel benthique favorisée par des activités anthropiques (pêche traditionnelle), (ii) les apports allochtones importants de matière comprenant des eaux usées, qui enrichissent la zone pélagique du lac (JUGNIA et SIME-NGANDO, 2001, JUGNIA et al., 1998, TADONLÉKÉ et al., 1998).

L'absence de corrélation entre les espèces zooplanctoniques et les variables physico-chimiques prises en compte, est une indication que la présence ainsi que les variations d'abondance de ces espèces au sein du lac municipal de Yaoundé, sont sous la dépendance d'autres facteurs parmi lesquels la station de prélèvement, la profondeur de prélèvement et les saisons. En effet, la variation horizontale expliquerait $3 \%$ de la variance totale des abondances des espèces étudiées, vraisemblablement à cause des espèces $B$. angularis et $K$. tropica qui se développent préférentiellement à la station II. II en est de même des $5 \%$ de la variance totale des abondances attribuable à la variation verticale, car les espèces $B$ angularis, $K$. tropica et $B$. falcatus sont apparemment inféodées aux eaux de la couche intermédiaire du lac 0,5 et $1 \mathrm{~m}$ ). Toutes ces espèces sont essentiellement pélagiques et la station II située au centre du lac tout comme les eaux de la couche intermédiaire, constitueraient des zones propices au développement de ces organismes microphages, probablement en raison non seulement de l'abondance des ressources, mais aussi du fait que ces zones offriraient, par leurs caractéristiques optiques et les changements transitoires au sein des principaux groupes fonctionnels d'organismes qui s'y effectuent, un refuge vis-à-vis, notamment, de la prédation (SAUNDERS et LEWIS, 1988, SIME-NGANDO et HARTMANN, 1991).

L'hétérogénéité dans les variations d'abondance des espèces étudiées s'est avérée plus prononcée d'un point de vue temporel (36\% de la variance totale), et l'ensemble des observations concernant les espèces les plus abondantes et fréquentes nous permet de supposer que les conditions environnementales qui affectent le développement potentiel des espèces et le déroulement des cycles biologiques, seraient dépendantes des deux saisons (la saison des pluies et la saison sèche) caractérisant les climats tropicaux, la période de transition entre ces deux saisons reste elle aussi une au cours de laquelle s'effectuent des variations.

La similarité entre les moyennes de données pluviométriques (1433 mm) et de température $\left(25 \pm 2,5^{\circ} \mathrm{C}\right)$ au cours de cette étude et celles caractérisant le climat dans la région du lac municipal de Yaoundé $\left(1576 \mathrm{~mm}\right.$ et $\left.24 \pm 2,5^{\circ} \mathrm{C}\right)$, indique que les conditions hydrologiques au cours de l'étude ont été proches des normales saisonnières. Or, dans ces conditions, la dynamique des espèces suivies est apparue importante au niveau de la couche intermédiaire des eaux du lac, mais aussi davantage influencée par les différentes saisons ainsi que les périodes de transition entre elles. Il s'agit là des considérations à prendre en compte dans le lac municipal de Yaoundé, lors des études faisant appel à la dynamique des populations de rotifères et cladocères ou d'inventaire faunistique de ces groupes à partir des prélèvements ponctuels. 


\section{REMERCIEMENTS}

Nous remercions R. POURRIOT de l'Université de Paris VI (France) et H. J. DUMONT de l'Université de Gand (Beigique) pour la lecture commentée et critique de la première version de ce manuscrit. Nous remercions également le personnel de l'ORSTOM (actuel IRD), et particulièrement J. BONVALLOT et M. MOLINIER pour l'assistance logistique lors des déplacements sur le terrain.

\section{RÉFÉRENCES BIBLIOGRAPHIQUES}

AKIMBUWA O. et ADENIYI I.F., 1996. Seasonal variation, distribution and interrelationship of Rotifers in OPA reservoir, Nigeria. Afr. J. Ecol., 34, 351-363.

ALMAZAN G. et BOYD C.E., 1978. An evaluation of Secchi disk visibility for estimating plankton density in fish ponds. Hydrobiologia, 61, 205-208.

AMOROS C., 1984. Crustacés Cladocères. Introduction pratique à la systématique des organismes des eaux continentales françaises. Bull. Mens. Soc. Lin. Lyon, $53,63$.

APHA, 1985. Standard methods for the examination of water and waste water, 16th ed. APHA, Washington DC.

ARCIFA M.S., 1984. Zooplankton composition of ten reservoirs in Southern Brazil. Hydrobiologia, 113, 137-145.

ARFI R. et PATRITI G., 1987. Impact d'une pollution urbaine sur la partie zooplanctonique d'un système néritique (Marseille - Cortiou). Hydrobiologia, 144, 11-23.

BENNDORF J. et HENNING M., 1989. Daphnia and toxic blooms of Microcystis aeruginosa in Bautzen Reservoir (GDR). Int. Rev. ges. Hydrobiol., 74, 233-248.

BLANCHER E.C., 1984. Zooplankton-trophic state relationships in some north and central Florida lakes. Hydrobiologia, 109, $251-263$.

BOPDA A., 1986. La dynamique de l'espace urbain à Yaoundé : Reconstruction et expansion post-coloniale du batiment In. Université de Yaoundé, Yaoundé, p. 250 .
BRANCO C.W.C., ROCHA M.I.A., PINTO G.F.S., GOMARA G.A. et DE FILIPPO R., 2002. Limnological features of funil Reservoir (R.J., Brazil) and indicator properties of rotifers and cladocerans of the zooplankton community. Lakes Reserv.: Res. and Manage, 7, 87-92.

CHIAMBENG G.Y., DUMONT H.J. et SEGERS H., 1991. Contribution to the knowledge of the zooplankton fauna of Cameroon: Some new records of Rotifera. Biol. Jb. Dodonaea, 59, 125-131.

CHIAMBENG G.Y., NJOCK J.C. et SEGERS H., 1994. Rotifera from south west, central and north provinces of Cameroon. Biol. Jb. Dodonaea, 61, 154-160.

CORBET S.A., GREEN J., GRIFFITH J. et BETNEY E., 1973. Ecological studies on crater lakes in West Cameroon lakes Kotto and Mboandong. J. Zool. Lond, 170, 309-324.

DUMONT H.J., 1977. Biotic factors in the population dynamics of rotifers. Arch. Hydrobiol., 8, 98-122.

DUMONT H.J., 1994. On the diversity of the Cladocera in the tropics. In "Studies in the ecology of tropical zooplancton" Dumont H.J., Green J. and Masundire H. (eds). pp. 27-28.

DUNCAN A. et GULATI R.D., 1981. Parakrama Samudra (Sri Lanka) project - a study of a tropical lake ecosystem, 3 . Composition, density and distribution of the zooplankton in 1979. Verh. Int. Ver. Limnol., 21, 1001-1008.

GANNON J.E. et STEMBERGER R.S., 1978. Zooplankton (specially crustaceans and 
rotifers) as indicators of water quality. Trans. amer. Micros. Soc., 97, 16-35.

GOPAL B. et WETZEL. R.G., 1995. Limnology in developing countries. International Scientific Publication, New Dehli, India.

GREEN J., 1972. Ecological studies on crater lakes in west Cameroon. Zooplankton of Barombi-mbo, Mboandong, lake Kotto and lake Soden. J. Zool. Lond., 166, 283-301.

GREEN J., 1994. The temperate-tropical gradient of planktonic Protozoa and Rotifera. Hydrobiologia, 272, 13-26.

GREEN J., CORBET A. et BETNEY E., 1974. Ecological studies on crater lakes in West Cameroon. Debunsha lake. $J$. Zool. Lond., 173, 199-223.

GREEN J. et KLING G.W., 1988. The genus Daphnia in Cameroon, West Africa. Hydrobiologia, 160, 257-261.

HABERMAN J., 1998. Zooplankton of lake Vörtjäv. Limnol., 28, 49-65.

JOSEE DE PAGGI S. \& KOSTE W.I., 1995. Addition to the checklist of Rotifers of the super order Monogononta recorded from Neotropis. Int. Revue ges. Hydrobiologia, 80, 133-140.

JUGNIA L.B. et SIME-NGANDO T., 2001. An assesment of the Mingoa stream input to the bacteriological quality of the Municipal lake of Yaoundé (Cameroon). Water Resour. Manag., 15, 203-209.

JUGNIA L.B., TADONLÉKÉ R.D., SIMENGANDO T., FOTO S.M. et KEMKA N., 1998. Short-term variations in the abundance and cell volume of bacterioplankton in an artificial tropical lake. Hydrobiologia, 385, 113-119.

KEMKA N., NJINE T., ZEBAZE T.S.H., NIYITEGEKA D., MONKIEDJE A., FOTO M.S., NOLA M. et COMPERE P., 2003. Quantitative importance of Cyanobacteria populations in a hypertrophic shallow lake in the subequatorial African region (Yaounde Municipal Lake, Cameroon). Arch. hydrobiol., 495-510.

KHAN M.A. et EJIKE C., 1984. Limnology and plankton periodicity of Jos Plateau Water Reservoir, Nigeria, West Africa. Hydrobiologia, 114, 189-199.

KLEIN L., 1959. River pollution: Chemical analysis. Butter worths, London.
KOSTE W., 1978. Rotatoria. Die Rädertiere Mitteleuropas begründet von Max Voigt. Gebrüder Borntraeger, Berlin.

LAIR N., REYES-MARCHANT P. et JACQUET V., 1998. Développement du phytoplancton, des ciliés et des rotifères sur deux sites de la Loire moyenne (France), en période d'étiage. Annl. Limnol., 34, 35-48.

LEGENDRE L. et WATT W.D., 1972. On a rapid technic for plankton enumeration. Annl. Inst. Oceanogr, XLVIII, 173-177.

LEWIS W.J., 1987. Tropical limnology. AnnI. Rev. Ecol. Syst., 18, 159-185.

LEWIS W.M.J., 2000. Basis for the protection an management of tropical lakes. Lakes \& Reservoirs: Research and Management, 5, 35-48.

MACKERETH F.T., HERON J. et TALLING J.F. (1978) Water analysis: some revised methods for limnologists. In: Sci. Publ. Freshwat. Biol. Assoc.England.

MAEMETS A., 1983. Rotifers as indicators of lake types in Estonia. Hydrobiologia, 104, 357-361.

MARGALEF R., 1983. Limnología. Omega, Barcelona.

MASUNDIRE H., 1989. Zooplankton composition and abundance in relation to water transparency and predation in Lake Kariba, Zimbabwe. Arch. Hydrobiol., 33, 513-520.

MATSUMURA-TUNDISI T., LEITÃO S.N., S. A.L. et MIYAHARA J., 1990. Eutrofização da Represa de Bara Bonita: Estrutura e organização da comunidade de Rotifera. Rev. Brazil. Biol., 50, 923-935.

NOGRADY T., 1983. Succession of planktonic rotifer populations in some lakes of the Eastern Rift Valley, Kenya. Hydrobiologia, 98, 45-54.

NOGUEIRA M.G., 2001. Zooplankton composition, dominance and abundance as indicators of enviromental compartmentalization in Jurumirim Reservoir (Paranapanema River), São Paulo, Brazil. Hydrobiologia, 455, 1-18.

NWADIARO C.S., 1987. Depth variation in the chemistry of Oguta lake in south Nigeria. Hydrobiol. Bull, 21, 133-140.

ORCUTT JR D. et PACE M.L., 1984. Seasonal dynamics of rotifer and crustacean zooplankton populations in a eutrophic, 
monemitic lake with a note on rotifer sampling techniques. Hydrobiologia, $119,73-80$.

PACE M.L., 1986. An empirical analysis: of zooplankton community size structure across lake trophic gradients. Limmol Oceanogr., 31, 45-55.

PAGGI J.C. et JOSE DE PAGGI S:, 1990 Zooplancton de ambientes lóticos e lênticos do Rio Parana Médió; Acta Limmol: Brasil., 3, 685-719.

POURRIOT R., 1968. Rotifères du lac Tchad. Bull. I.F.A.N., X×X, 471-496.

POURRIOT R. et FRANCEZ A.J., 1986. Rotifères. Introduction pratique à la systématique des: organismes des eaux continentales françaises. Bull. mens. Soc. Lin. Lyon, 55, 37.

SAUNDERS I.J.F. et LEWIS W.M.J., 1988. Compositionand seasonality of the zooplankton community of lake Valencia, Venezuela. J. Plank. Res., 10, 957-985.

SEGERS H. et MERTENS J., 1997. New Rotifera from the Korup National Park, Cameroon. J. of Nat. Hist., 31, 663-668.

SHIEL R.J., 1995. A guide to identification of rotifers, cladocerans and copepods from Australian Inland water. CRCFE Ident. Guide 3, p 144.

SIME-NGANDO T. et HARTMANN H.J., 1991. Short-term variations of the abundance and biomass of planktonic ciliates in a eutrophic lake. Europ. J. protistol., $27,249-263$.

SLADECEK V., 1983. Rotifers as indicators of water quality. Hydrobiologia, 100, 169-201.
SMIRNOV N:N:, 1996: Chydoridae. Guide to the identification of the microinvertebrate of: the continental waters of the world. S.P.B., The Hague.

SMIRNOV N.N. et KOROVCHINSKY M., 1995. Introduction to the cladocera. ITC, 157.

SUCHEL B., 1987. Les climats du Cameroun. In Bordeaux HI, Bordeaux, $p$. 1,186 .

TADONLÉKÉ R.D., JUGNIA L.B., SIMENGANDO T., ZÉBAZE T.S.H. et NOLA M., 1998. Short-term vertical distribution of phytoplankton populations in a shallow tropical! Iake (Lake Municipal Yaoundé, Cammeroon) Afch Hydrobiol., $143,469-296$ :

THIOULOUSE J, CHESSEC D, DOLEDEC S. et OLIVIER JM/, 1997. ADE-4: a multivariate analysis sandcgraphical display softwares. Statistics sand Computing, 7, 75-83.

THOUVENOT A, DEBRYAS D., RICHARDOT $M$, et DEVAUXJ, 1999. Bacterivory of metazooptankiton, ciliates: and flagellates in a newly flooded reservoir. J. Plankton Res: 21, 1659;1679.

VINER A.B., 1969: The chemistry of the water of lake George, Uganda. Verth. Int. Verein. Limnol., 17, 289-296.

WYLIE J.L. ot CURRIE D.J., 1991. The relar tive impontance of bacteria and algae as food source for crustaceani zooplankton. Limnol. Oceanogr., 36, 708-728.

$$
\begin{aligned}
& \text { tै } \\
& 4 \ldots \\
& 631 \% \\
& \because z=\ldots \quad \bar{b}
\end{aligned}
$$

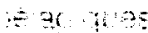

$$
\begin{aligned}
& \text { It: } \\
& +3
\end{aligned}
$$

